\title{
Seismic constraints on open clusters
}

\author{
L. Piau ${ }^{1}$, J. Ballot ${ }^{2}$, and S. Turck-Chièze ${ }^{2}$ \\ ${ }^{1}$ Institut d'Astronomie et d'Astrophysique, ULB, CP226, 1050 Brussels, Belgium \\ e-mail: piau@astro.ulb.ac.be \\ 2 CEA/DSM/DAPNIA/Service d'Astrophysique, CE Saclay, 91191 Gif-sur-Yvette Cedex 01, France \\ e-mail: [jballot; cturck]@cea.fr
}

Received 3 June 2004 / Accepted 27 August 2004

\begin{abstract}
The aim of this theoretical and modelling paper is to derive knowledge on the global and structural parameters of low-mass stars using asteroseismology and taking advantage of the stellar collective behavior within open clusters. We build stellar models and compute the seismic signal expected from main sequence objects in the $0.8-1.6 M_{\odot}$ range. We first evaluate apparent magnitudes and oscillations-induced luminosity fluctuations expected in the Hyades, the Pleiades and the $\alpha$ Persei clusters. The closest cluster presents a feasible challenge to observational asteroseismology in the present and near future. The remainder of the work therefore focuses on the Hyades.

We combine seismological and classical computations to address three questions: what can be inferred about 1) mass; 2) composition; and 3) extension of outer convection zones of solar analogs in the Hyades. The first issue relies on the strong sensitivity of the large separation to mass. We show that seismic constraints provide masses to a precision level $\left(0.05 M_{\odot}\right)$ that is competitive with the actual mass estimations from binary systems. Then large separations $(\Delta v)$ and second differences $\left(\delta_{2} v\right)$ are used to respectively constrain metal and helium fractions in the Hyades. When plotted for several masses, the relation of effective temperature $\left(T_{\text {eff }}\right)$ vs. large separation $(\Delta v)$ is found to be strongly dependent on the metal content. Besides this the second difference main modulation is related to the second ionization of helium. An accuracy in the helium mass fraction of 0.02 to 0.01 can be achieved provided mass and age are accurately known, which is the case for a few Hyades binary systems. The second difference modulations are also partly due to the discontinuity in stellar stratification at the convective envelope/radiative core transition. They permit direct insight in the stellar structure. We compute acoustic radii of the convective bases for different values of the mixing length theory parameter $\alpha_{\mathrm{MLT}}$ in convection modelling, i.e. different convective efficiency in the superadiabatic layers. For a given effective temperature we show that the acoustic radius changes with convection efficiency. This suggests that seismology can provide constraints on the extension of outer convection and also more generally on the direct approaches of convection and dynamical phenomena being currently developed.
\end{abstract}

Key words. Galaxy: open clusters and associations: general - stars: oscillations - stars: late-type - stars: abundances stars: interiors

\section{Introduction}

Helioseismology achieved many successes in allowing direct determinations of the depth of the solar convection zone (Christensen-Dalsgaard et al. 1991) or photospheric mass fraction of helium (Basu \& Antia 1995). In a comparable fashion the development of space-borne asteroseismic experiments within the coming years and the increasing number of groundbased observations should yield extremely precious data on other stars. In this paper we discuss some aspects of the possible contribution of asteroseismology to the determination of mass, composition and structure of solar-like stars. We address the Hyades, Pleiades and $\alpha$ Persei open clusters. These clusters have been extensively studied from theoretical and observational viewpoints (see Lebreton 2000, for a recent review) and their stars are among the targets of future asteroseismic missions such as EDDINGTON (Favata et al. 2000). Some of them will also be interesting targets for the recent and promising instruments HARPS (Pepe et al. 2003) or ESPaDOnS (Donati et al. 2004). Contrary to field stars they offer the possibility to combine observations for stars having similar ages and compositions. In consequence they make it possible to exploit collective effects in seismic and stellar properties of stars with different masses. Finally they span a range of age and composition that should translate into significant differences in the seismic signals of solar-like stars.

We compute stellar models and subsequently use them to predict oscillation frequencies. We use the CESAM code (Morel 1997) for pre-main sequence and main sequence modelling. Above $5800 \mathrm{~K}$ our version of the code relies on the OPAL2000 equation of state (Rogers et al. 1996) and opacities (Iglesias \& Rogers 1996). For lower temperatures the OPAL equation of state is replaced by the MHD equation of state (Mihalas et al. 1988) and the opacities are from 
Table 1. Apparent visual magnitude $m_{V}$ and expected luminosity fluctuations for $0.78,0.8,0.95,1,1.15,1.3,1.5$ and $1.6 M_{\odot}$ stellar models. Assumed ages are 90, 125 and $625 \mathrm{Myr}$ for $\alpha$ Persei, Pleiades and Hyades respectively. We consider non-solar repartition of metals in the Hyades and $Y=0.26$ (see Sect. 3). For the two other clusters we assume solar composition. Luminosity fluctuations are probably underestimated for the 1.5 and $1.6 M_{\odot}$ models. Rows 4 to 7 list various seismological features for the age and composition of the Hyades.

\begin{tabular}{lcccccccc}
\hline \hline Mass $M_{\odot}$ & 0.78 & 0.8 & 0.95 & 1 & 1.15 & 1.3 & 1.5 & 1.6 \\
\hline$m_{V}$ (Pleiades) & 12.1 & 12.0 & 10.9 & 10.6 & 9.8 & 9.1 & 8.4 & 8.1 \\
\hline$m_{V}(\alpha$ Persei) & 13.1 & 12.9 & 11.8 & 11.5 & 10.7 & 10.1 & 9.3 & 9.1 \\
\hline$m_{V}$ (Hyades) & 10.1 & 9.9 & 8.8 & 8.5 & 7.7 & 7.0 & 6.3 & 6.0 \\
Luminosity fluctuations $(\mathrm{ppm})$ & 1.8 & 1.9 & 2.9 & 3.2 & 4.6 & 6.2 & 8.7 & 9.9 \\
Frequency of maximum amplitude $v_{\max }(\mathrm{mHz})$ & 3.5 & 3.5 & 3.9 & 3.9 & 3.7 & 3.3 & 2.7 & 0.8 \\
Cut-off frequency $v_{\mathrm{c}}(\mathrm{mHz})$ & 8.7 & 8.5 & 6.9 & 6.3 & 4.8 & 3.6 & 2.4 & 2.1 \\
\hline
\end{tabular}

Alexander \& Ferguson (1994). The atmosphere is restored using Hopf's law (Mihalas 1978). It is connected to the envelope at optical depth 10 where the diffusion approximation for radiative transfer becomes valid (Morel et al. 1994). Microscopic diffusion is always taken into account following the Michaud \& Proffitt (1993) prescriptions. Finally the convection zones are assumed to be fully homogeneous, and modeled using the mixing-length theory (hereafter MLT) in a formalism close to that of Böhm-Vitense (1958). Unless mentioned explicitly we consider $\alpha_{\mathrm{MLT}}=1.766$ which is our Sun-calibrated value. No overshooting has been considered in convective cores or outer convective regions. Once the stellar structure has been obtained we use the adiabatic pulsation package of Jørgen Christensen-Dalsgaard (1982) to compute oscillation frequen$\operatorname{cies}^{1}$. As usual $n$ and $\ell$ denote respectively the radial order and the degree of the mode whose frequency is written as $v_{\ell, n}$.

Before we present the approaches we used to relate the stellar parameters to the seismic signal we address the question of the mode amplitudes in the following section. This question is a crucial one for the efficiency of future seismic campaigns.

In the Sect. 3 we focus on the impact of global parameters on the asteroseismic data. Both initial mass and chemical composition are important in stellar evolution. Helium is the second most abundant constituent of the Universe. It is is however impossible to determine the helium fraction from spectral analysis of low-mass stars. Its abundance therefore remains undetermined in all open clusters except the Hyades. In this open cluster lower-main sequence fits that constrain helium content have been performed (Perryman et al. 1998; Pinsonneault et al. 1998). Stellar mass determination based on close binaries is similarly a difficult task. Except in the case of nearby systems such as $\alpha$ Cen the uncertainty in the mass generally is $>0.1 M_{\odot}$ (Söderhjelm 1999). Addressing the specific case of the Hyades cluster we show what improvements seismology could give regarding the helium mass fraction and more generally composition.

In Sect. 4 we discuss the seismic characterisation of the transition from the radiative interior to the convective envelope in solar-type stars. Then we evaluate the impact of changes in

\footnotetext{
1 Aarhus Adiabatic Pulsation Package, presently available at http://astro.phys.au.dk/ jcd/adipack.n/
}

the convective efficiency in the superadiabatic regime over the depth of the convection zone. We therefore propose seismology as a tool to calibrate the $\alpha_{\text {MLT }}$ parameter or more importantly the convection efficiency to be inferred from direct hydrodynamic computations for different surface conditions $\left(T_{\mathrm{eff}}\right.$, $\log g$ ).

A discussion of our results and our conclusions are given in Sect. 5 .

\section{Detectability of oscillations}

The detection and study of solar-like oscillations modes are restricted by several factors. Not only are they limited to a certain range of visual apparent magnitude but they also depend on the amplitude of the luminosity modulations. In this section we discuss the anticipated amplitude of the p-modes for the various stars we modeled. This is a central point in the perspective of space asteroseismic missions such as COROT (Baglin et al. 2002) and MOST (Walker et al. 2003) or ground based observations. We have derived the apparent magnitudes in $\mathrm{V}$ band on the $\sim 0.77$ to $1.6 M_{\odot}$ stellar mass range and for the three open clusters (Table 1). We take $M_{\mathrm{Bol}}=4.72-2.5 \log _{10}\left(L / L_{\odot}\right)$ (ie $M_{\mathrm{Bol} \odot}=4.72$ ) and we apply the bolometric correction $B C_{V}$ as deduced from a bilinear interpolation in $T_{\text {eff }}$ and $\log g$ tables provided in Houdashelt et al. (2000) $)^{2}$ where $T_{\text {eff }}$ is the effective temperature and $\log g$ the $\log$ of the surface gravity. We have used distance moduli based on HIPPARCOS parallaxes. We take $m-M=5.37 \pm 0.07$ and $6.31 \pm 0.08$ respectively for the Pleiades and the $\alpha$ Persei cluster (van Leeuwen 1999). For the Hyades we assume $m-M=3.33 \pm 0.01$ (Perryman et al. 1998). The extinction $A_{\mathrm{v}}$ is negligible for the Hyades, close to $0.04 \mathrm{mag}$ for the Pleiades and $0.1 \mathrm{mag}$ for $\alpha$ Persei (Pinsonneault et al. 1998). Owing to these values no reddening has been taken into account in the process of computing visual apparent magnitudes from the absolute bolometric magnitudes provided by the models. The magnitudes we report are therefore only accurate to $0.1 \mathrm{mag}$.

${ }^{2}$ IAU Commission 25 (1997) XIII General Assembly recommended the bolometric magnitude of the Sun to be defined as +4.75 . However, following Houdashelt et al. (2000) we use +4.72 for consistency. 
Let us now focus on the expected amplitudes for the modes. These are determined by the relative efficiencies of the driving and damping processes. For solar analogs the most probable explanation of driving of modes consists of the fluctuations of the Reynolds stress associated with convection in the superadiabatic layers. In stellar conditions the acoustic emission increases as $M_{\mathrm{t}}^{7.5}$ where $M_{\mathrm{t}}$ is the turbulent Mach number (Goldreich \& Kumar 1990). Recent computations suggest that $M_{\mathrm{t}}$ reaches a maximum around $1.6 M_{\odot}$ on the zero age main sequence (ZAMS) and so does the oscillation amplitude (Houdek et al. 1999). The most massive stars we address here are therefore expected to have the largest fluctuation amplitudes. In terms of surface velocity, the observations suggest:

$v_{\text {osc }} \propto\left(L_{\star} / M_{\star}\right)^{0.7}$

In this relation, as in the following ones, $L_{\star}$ and $M_{\star}$ stand for stellar bolometric luminosity and mass in solar units respectively. Kjeldsen \& Bedding (1995) and Houdek et al. (1999) have proposed similar laws with somewhat higher exponents. However they predict large velocity fluctuations that exceed the actual observations in the case of most massive stars such as Procyon $\left(1.46 M_{\odot}\right)$. Using theoretical models of stochastic excitation (Samadi \& Goupil 2001), Samadi et al. (2004) confirm this power-law dependence with an almost identical exponent (0.8 instead of 0.7). Space-borne experiments will measure brightness modulations into which $v_{\text {osc }}$ has to be converted. Such an analysis is performed in Kjeldsen \& Bedding (1995) and we will not reproduce it here. As the main result of the computations one obtains $\left(\frac{\delta L}{L}\right)_{\text {Bol }} \propto \frac{v_{\text {osc }}}{\sqrt{T_{\text {eff }}}}$ which together with Eq. (1) yields an estimate of the dependence of the amplitude of the luminosity fluctuations on fundamental stellar parameters:

$\left(\frac{\delta L}{L}\right)_{\mathrm{Bol}} \propto \frac{1}{\sqrt{T_{\mathrm{eff}}}}\left(\frac{L_{\star}}{M_{\star}}\right)^{0.7}$.

If we now use the Sun as a calibrator, this simple law provides an estimation of the luminosity variations induced by oscillations. Such an assumption should be adequate, for the stars we consider have solar-like (convectively powered) oscillations and the external convection properties should vary smoothly with stellar type. The oscillations associated with solar luminosity fluctuations are of the order of $4 \mathrm{ppm}$ (Kjeldsen $\&$ Bedding 1995). Therefore if we express the stellar mass and luminosity in solar units we finally deduce:

$\left(\frac{\delta L}{L}\right)_{\mathrm{Bol}}=4 \times \sqrt{\frac{5780}{T_{\mathrm{eff}}}}\left(\frac{L_{\star}}{M_{\star}}\right)^{0.7}$.

Using this last equation, Table 1 gives the amplitude of the luminosity fluctuations as a function of mass for the Hyades models. It also indicates the magnitudes of the corresponding objects in the other two clusters. Because of differences in composition and evolutionary stage, the relative amplitudes of the fluctuations decrease slightly at a given mass when going from Hyades to the younger clusters. We do not compute any metallicity impact on the amplitudes. The small variations are instead most probably due to an age effect which is expected to be very small for $\sim 0.5$ Gyr difference (Houdek et al. 1999) and we do not reproduce them here in detail. The maximum variation concerns the most massive star where it reaches $0.6 \mathrm{ppm}$ i.e. $\approx 6 \%$.

Also of interest is the range of frequencies for which solarlike p-modes oscillations should be the best visible. This frequency range roughly covers a region from the frequency $v_{\max }$ where the largest amplitude is expected up to the cutoff frequency $v_{\mathrm{c}}$ and a similar frequency extent below $v_{\max }$. We provide $v_{\max }$ in Table 1 together with the atmospheric cut-off frequency $v_{\mathrm{c}} ; v_{\mathrm{c}}$ is computed from its usual expression $2 \pi v_{\mathrm{c}}=\frac{c_{\mathrm{s}}}{2 H_{\mathrm{p}}}$ with $c_{\mathrm{s}}$ and $H_{\mathrm{p}}$ respectively the sound speed and pressure scale height in the atmosphere; $v_{\max }$ is estimated following $v_{\max }=$ $\frac{v_{\max }}{H_{\mathrm{p}}}$ where $v_{\max }$ stands for the maximum convective velocity in the superadiabatic layers and $H_{\mathrm{p}}$ is the corresponding pressure scale height. Because of the sharp drop in density and temperature on the one hand and the requirement for nearly constant convective flux on the other hand, convective motions are substantially accelerated in the upper superadiabatic layers. The driving of solar-like oscillations is highly sensitive to the kinetic energies of convective eddies. Thus its efficacy reaches a maximum in the upper superadiabatic layers. Modes of frequencies $v_{\max }$ similar to the local turn-over frequency $v_{\text {to }}$ where the convective velocity is maximal exhibit the largest oscillation amplitudes and therefore $v_{\max } \approx v_{\text {to }}$. For modes having $v_{\text {osc }}>v_{\text {to }}$ the excitation vanishes because of a lack of coherence between eddies at higher frequencies; while in contrast for $v_{\text {osc }}<v_{\text {to }}$ there is a rough equipartition between convective kinetic and mode energies (Goldreich \& Keeley 1977). However because of the increase of the inertia of the modes the amplitudes diminish with the decrease of $v_{\text {osc }}$. We made a rough estimate for the convective velocity $v$ using the mixing length theory formulae (precise demonstrations are to be found in text books):

$$
\begin{aligned}
& v=\frac{\alpha_{\mathrm{MLT}}}{2} \sqrt{\frac{1}{2} g \delta H_{\mathrm{p}}} \frac{\Gamma}{B^{1 / 2}} \\
& B=\frac{1}{162} \frac{\left(\alpha_{\mathrm{MLT}} H_{\mathrm{p}}\right)^{4}\left(\rho C_{\mathrm{p}}\right)^{2} \delta g}{K^{2} H_{\mathrm{p}}}
\end{aligned}
$$

where the various symbols have their usual meanings: $\alpha_{\mathrm{MLT}}$ is the mixing length parameter; $g$ is the local gravity; $\delta=$ $-\left(\frac{d \ln \rho}{d \ln T}\right)_{P} ; \Gamma$ is the convective efficiency; $C_{\mathrm{p}}$ is the gas specific heat at constant pressure; $\rho$ and $K$ are respectively the gas density and thermal conductivity. These various quantities are derived from our models. We use the Eq. (4) as a scaling law considering that in the case of the Sun the maximum oscillation amplitude is at $3.3 \mathrm{mHz}$ which corresponds to the well known 5-min oscillations. Our solar reference model for convection velocities is the calibrated "Btz" model from Brun, Turck-Chièze \& Zahn (1999) for which the Eq. (4) provides a maximal velocity $v_{\max }=2.6 \mathrm{~km} \mathrm{~s}^{-1}$.

The magnitudes and oscillation amplitudes computed here are indicative of the observational accuracy needed to address solar objects in nearby open clusters using seismology. Even though the COROT space mission does not primarily aim at clusters we will consider its performance as indicative of what is now within technical reach. For a $m_{V}=9$ mag the threshold of oscillation detection expected for this mission is 
$\left(\frac{\delta L}{L}\right)_{\text {Bol }}=2.4 \mathrm{ppm}$ (Baglin et al. 2001). Table 1 therefore suggests that Hyades stars around and above solar mass should become accessible to seismic measurements in the near future. A Hyades star of $0.95 M_{\odot}$ is for instance interesting in several respects. It should exhibit $m_{V}$ in the 8.7 to 9.1 range depending on the actual age and composition of the Hyades (see Table 3 of Sect. 3). This somewhat high magnitude is however compensated by a $\left(\frac{\delta L}{L}\right)_{\text {Bol }}$ we expect to be above $2.8 \mathrm{ppm}$. In the next section we develop the informations on mass one could extract from such a seismic target. For the Pleiades and $\alpha$ Persei the situation is far less favourable because of their distances. With $1.3 M_{\odot}$ and $1.6 M_{\odot}$ stars having a visual magnitude of $\approx 9$ in these respective clusters, solar mass stars will be difficult targets there. The oscillation amplitudes expected above $1.3 M_{\odot}$ are sensibly higher than solar ones and render corresponding stars better targets. One nevertheless needs to be cautious concerning the granulation noise which may limit the observations of luminosity fluctuations. We end this section with two remarks: 1) Because of its youth a Hyades solar analog has a slightly larger magnetic and chromospheric activity than our Sun (see Paulson et al. 2002 for recent evaluations). This could be problematic for the clear detection and and interpretation of the oscillatory modes. 2) Stars having masses between 1.5 and $1.6 M_{\odot}$ are $\delta$ Scuti variables when they are near to the ZAMS. We are presently not interested in the difficult question of low frequency modes excited by radiative processes. In order to avoid such modes we shall limit ourselves in subsequent computations to modes of orders above $n=10$ for which the $\kappa$ mechanism has been shown to be inefficient (Michel et al. 1999). We note that both radiatively and convectively driven oscillations are expected in $\delta$ Scuti variables. Furthermore because the regions of $\delta$ Scuti involved in the driving of the modes are not at dynamical equilibrium the expected luminosity fluctuations $\frac{\delta L}{L}$ are much higher than predicted when using Eq. (4) (see Samadi et al. 2002).

\section{Global parameters and seismology in the Hyades}

\subsection{Composition determination}

Asteroseismology may complement spectroscopic chemical determinations. It will provide some indications on composition as, because of opacity effects, metals and helium have a direct impact on stellar global parameters (luminosity, effective temperature) and structure. This information is of course dependent on the various assumptions made in the modelling (convection parameter, diffusion processes, age....) ${ }^{3}$.

As a first step, we will exploit the collective seismic behaviours of stars of similar age and composition. In open clusters it is indeed possible to draw conclusions from seismic HR diagrams in the same way as from the classical HR diagram. Figure 1 is a transposition of the usual theoretical HR diagram where bolometric luminosity has been replaced by the large

\footnotetext{
${ }^{3}$ The past year's developments concerning solar surface oxygen fraction however reminds us that spectroscopic measurements also are strongly dependent on models (Asplund et al. 2004).
}

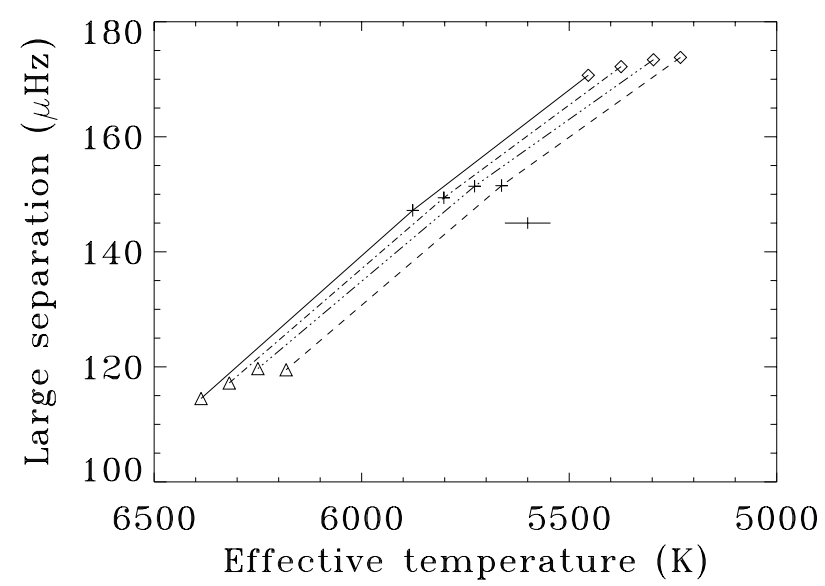

Fig. 1. Large separation as function of effective temperature between $\sim 5000$ and $6500 \mathrm{~K}$ for varying possible composition model of the Hyades. The solid line and dashed line correspond to $\mathrm{C} 1([\mathrm{Fe} / \mathrm{H}] \neq$ $[\mathrm{O} / \mathrm{H}], Y=0.26)$ and $\mathrm{C} 5([\mathrm{Fe} / \mathrm{H}]=[\mathrm{O} / \mathrm{H}], Y=0.26)$ respectively. For the same value of the abscissa they are separated by $\sim 15 \mu \mathrm{Hz}$. The dot-dashed line and the triple dot-dashed line represent $\mathrm{C} 2([\mathrm{Fe} / \mathrm{H}] \neq$ $[\mathrm{O} / \mathrm{H}], Y=0.25)$ and $\mathrm{C} 3([\mathrm{Fe} / \mathrm{H}] \neq[\mathrm{O} / \mathrm{H}], Y=0.24)$ respectively. These models show that similarly to the usual HR diagram a decrease of the helium fraction has the same effect as the increase of the metal fraction. The diamonds, crosses and triangles are the positions of the 0.95, 1.07 and $1.25 M_{\odot}$ models for each composition. The horizontal error bar below the curves at $\approx 5600 \mathrm{~K}$ shows the typical uncertainty of the effective temperature. We also indicated a $1 \mu \mathrm{Hz}$ wide vertical bar. The expected precision for mode frequency determination from EDDINGTON is $0.3 \mu \mathrm{Hz}$.

Table 2. The five composition considered for the Hyades.

\begin{tabular}{llllll}
\hline \hline & C1 & C2 & C3 & C4 & C5 \\
\hline Helium mass fraction & 0.26 & 0.25 & 0.24 & 0.28 & 0.26 \\
{$[\mathrm{Fe} / \mathrm{H}]^{a}$ (dex) } & 0.127 & 0.127 & 0.127 & 0.127 & 0.127 \\
{$[\mathrm{O} / \mathrm{H}]^{a}$ (dex) } & -0.07 & -0.07 & -0.07 & -0.07 & 0.127 \\
\hline
\end{tabular}

${ }^{a}[\mathrm{Fe} / \mathrm{H}]$ represents iron and iron-group metals while $[\mathrm{O} / \mathrm{H}]$ represents oxygen and non-iron-group metals.

separation. This figure therefore does not show the traditional seismic HR diagram in which the small separation (defined as $\left.\delta v_{\ell, n}=v_{\ell, n}-v_{\ell+2, n-1}\right)$ is plotted as a function of the large separation (defined as $\Delta v_{\ell, n}=v_{\ell, n}-v_{\ell, n-1}$ ) (Christensen-Dalsgaard 1988). In this study we have considered five plausible compositions for the Hyades, summarized in Table 2. The first composition (hereafter $\mathrm{C} 1$ ) assumes that $[\mathrm{Fe} / \mathrm{H}]=0.127 \mathrm{dex}$ (Boesgaard \& Friel 1990) applies to all iron-group metals we consider ${ }^{4}$ but uses $[\mathrm{O} / \mathrm{H}]=-0.07$ (Garcia Lopez et al. 1993) for oxygen and other metals. It therefore presents a non-solarfraction repartition among metals. For this $\mathrm{C} 1$ composition we choose a helium fraction $Y=0.26$ as supported by fitting of the lower main sequence (Perryman et al. 1998) and by massluminosity relation of binaries (Lebreton et al. 2001). The second (C2), third (C3), and fourth composition (C4) only differ from $\mathrm{C} 1$ through $Y=0.25 Y=0.24$ and $Y=0.28$ respectively. The fifth composition (C5) assumes $[\mathrm{Fe} / \mathrm{H}]=0.127 \mathrm{dex}$,

\footnotetext{
${ }^{4} \mathrm{Ni}, \mathrm{Fe}, \mathrm{Mn}, \mathrm{Cr}$, Ti.
} 
$Y=0.26$ and (the usual) solar repartition among metals. Provided that the effective temperature is reliably determined, Fig. 1 illustrates that it should be possible to make a clear distinction between $\mathrm{C} 1$ and $\mathrm{C} 5$ : in the 5000 to $6000 \mathrm{~K}$ effective temperature range the mean large separation $\Delta v$ (see Sect. 3.2 for the precise definition) varies by $\sim 10 \mu \mathrm{Hz}$ between a metalrich Hyades star model (considering that the metal fractions follow the $[\mathrm{Fe} / \mathrm{H}]=0.13$ dex value) and its metal-poor counterpart (considering $[\mathrm{O} / \mathrm{H}]=-0.07$ dex as Garcia Lopez et al. (1993) suggests) that exhibits the same effective temperature. Such a difference is therefore quite significant when translated to expected accurracies of seismic experiments. The typical uncertainty in effective temperature found in the Hyades lowmass stars is $55 \mathrm{~K}$ (Thorburn et al. 1993) whereas the large separation determination should be affected by an error around $0.1 \mu \mathrm{Hz}$. The temperature uncertainties therefore have a much larger impact on the interpretation of Fig. 1 in terms of composition of the Hyades.

It is worth estimating the limits of this method. Although the main sequences in Fig. 1 strongly depend on composition caution is needed when interpreting their positions in these terms. The large separation is directly fixed by the radius and consequently by the assumptions about convection modeling. For instance for a Hyades $\mathrm{C} 1$ composition and $0.95 M_{\odot}$ model a change of $\alpha_{\mathrm{MLT}}$ from our standard value 1.76 to 1.56 induces decreases of $70 \mathrm{~K}$ and $4 \mu \mathrm{Hz}$ in $T_{\text {eff }}$ and mean $\Delta v$ respectively. These variations of convective efficacy remain plausible (see Sect. 4). Let us now compare the accuracy of the determination of the composition from our seismic HR diagram with the precision of the classical HR diagram. The smaller the uncertainties in the large separation (respectively the luminosity) the better defined the main sequence and the more precise the composition determination through a fit of the lower main sequence or the age determination from the turn-off position. In the framework of usual HR diagram analysis, Stello \& Nissen (2001) establish that the mean error in absolute visual magnitude can be expressed as $\sigma\left(M_{V}\right)=\left[12 \sigma(b-y)^{2}+\left(2.17 \frac{\sigma(\pi)}{\pi}\right)^{2}\right]^{1 / 2}$, where $b$ and $y$ are the color bands from the Strömgren ubvy photometry while $\pi$ is the parallax. The precision in $M_{V}$ for individual members of clusters is mainly affected by uncertainties in these quantities. In the case of the Pleiades and the $\alpha$ Persei clusters the HIPPARCOS value for $\frac{\sigma(\pi)}{\pi}$ of the brightest stars exceeds 0.1 (Eggen 1998). On the other hand Stello \& Nissen (2001) estimate $\sigma(b-y) \approx 0.01$. The global error in the absolute magnitude $M_{V}$ of individual stars in the Pleiades and $\alpha$ Persei therefore is $>\left(0.12^{2}+0.21^{2}\right)^{1 / 2} \approx 0.25$. In Fig. 2 we illustrate and compare the impact of the composition on positions of the main sequence in a classical and in the seismic HR diagram. We consider three plausible compositions for the Pleiades that present variations of $\Delta Y=0.1$ and $\Delta[\mathrm{Fe} / \mathrm{H}]=0.05 \mathrm{dex}$ among each other. We remark that with respect to the errors the separation along the ordinate axis is much larger in the seismic than in the classical HR diagram.

Recently de Bruijne et al. (2001) derived extremely accurate individual Hyades distances making use of HIPPARCOS secular parallaxes. This brings the accuracy in the absolute
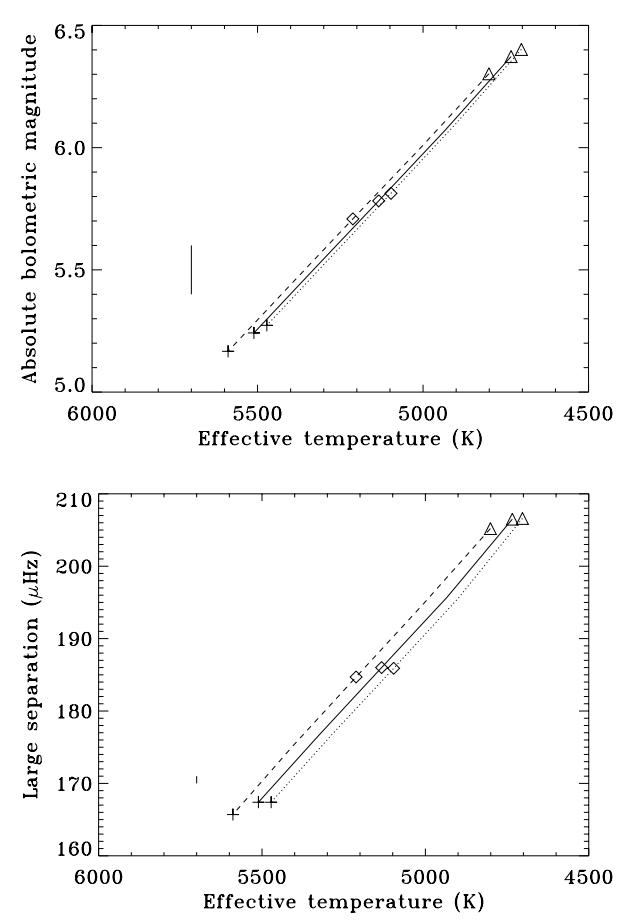

Fig. 2. Upper panel: usual theoretical HR diagram for Pleiades. Continuous line: solar composition stars $(X=0.7354, Y=0.2465)$; Dashed line: helium fraction is increased by 0.1 but $[\mathrm{Z} / \mathrm{H}]$ is kept unchanged so that $X=0.7256, Y=0.2565$. Dotted line: $[\mathrm{Z} / \mathrm{H}]=$ 0.05 dex with respect to solar composition so that $X=0.7275$, $Y=0.2524$. The vertical error bar $(0.2 \mathrm{mag})$ is representative of the absolute magnitude uncertainty for the Pleiades cluster. Triangles, diamonds, and crosses show $0.8,0.9$ and $1 M_{\odot}$ stars respectively. Lower panel: seismic HR diagram for the same cluster: Large separation vs. effective temperature. The plot conventions are similar. The minute vertical error bar of $1 \mu \mathrm{Hz}$ is representative of the maximal uncertainty in the large separation. We note that the separation between main sequences is much larger in the seismic diagram.

magnitudes $M_{V}$ down to the $\sim 0.05$ level and allowed the authors to produce an unprecedentedly narrow main sequence. $M_{V}$ is however not a quantity that is provided through stellar structure computation and that can be used in a theoretical HR diagram. We note that the $B-V$ to $T_{\text {eff }}$ calibration is still controversial (Bessell et al. 1998, Lejeune et al. 1998) and is found to be inappropriate below $\sim 5000 \mathrm{~K}$ (de Bruijne et al. 2001). Different transformations lead to zero age main sequences varying by up to $0.2 \mathrm{mag}$ in the $M_{V}, B-V$ plane and between $B-V=0.7-0.8$ (ie $T_{\text {eff }} \sim[5500,5200] \mathrm{K}$ ) whereas they are similar in the $M_{\mathrm{Bol}}, T_{\text {eff }}$ plane (Robichon et al. 1999). Moreover the $M_{V}$ transformation to bolometric luminosity is subject to the difficult question of bolometric corrections $\left(B C_{V}\right) . B C_{V}$ depends on the effective temperature and also critically depends on metallicity (Alonso et al. 1996) and surface gravity (Arribas \& Martinez-Roger 1988). We consequently stress here that as long as detailed metal abundances remain uncertain absolute luminosity determination might be biased. In view of this different sources of uncertainty we consider that seismic diagrams could give valuable insight into composition even for the nearest open cluster. 
In Figs. 1 and 2 the main sequences are not surprisingly dependent on the helium fraction. Slight opposite variations in the helium or metal fractions have similar impact on stellar luminosity and effective temperature so that a variation of the helium fraction or metal fraction do not seem to be a priori distinguishable. Hence main sequence fitting can only lead to metal abundances once the helium fraction is known (and conversely). We nevertheless point out that if the Hyades metal fraction is around -0.07 dex instead of 0.1 dex then the main sequence clearly lies above this last metal-rich main sequence for any possible helium fraction: the $\mathrm{C} 3$ composition main sequence is in a sense a limit that can be assigned to metal-poor main sequences for it has $Y=0.24$ which is critically close to the actual primordial helium evaluations: Izotov \& Thuan (1998) propose $Y_{\mathrm{p}}=0.245 \pm 0.004$ and Peimbert \& Peimbert (2000) propose $Y_{\mathrm{p}}=0.2345 \pm 0.003$.

In order to specifically check for the helium effects we have used the second difference: $\delta_{2} v_{\ell, n}=v_{\ell, n-1}-2 v_{\ell, n}+v_{\ell, n+1}$. As is well known, the fluctuations of $\delta_{2} v_{\ell, n}$ with frequency outline the presence of two rapid variations in the stellar structure: firstly the region of the second ionization of helium, secondly the discontinuity at the base of the outer convection zone. In a recent work Basu et al. (2004) suggest that it is possible to determine the helium fraction Y using the mean amplitude of the second difference modulation provided that both mass and radius are accurately known. In that work the signature appears more clearly around $1.2 M_{\odot}$ (see also Lopes et al. 1997). Masses are generally not known in the Hyades and there are no measurements of radii. However the age is known and some binary systems such as Finsen 342 have their masses precisely determined. Because of its mass $\left(1.25 M_{\odot}\right)$ and luminosity $\left(m_{V} \approx 7.4\right)$ Finsen $342 \mathrm{~B}$ should be an interesting target for helium determination. We have built models of this $\operatorname{star}^{5}$ and computed the second differences for different initial helium $Y$ values. Assuming that the second ionization of helium acts as a discontinuity, the second difference was fitted to the form ${ }^{6}$ :

$$
\begin{aligned}
\delta_{2} v= & \left(a_{1}+a_{2} v+\frac{a_{3}}{v^{2}}\right) \\
& +\left(b_{1}+\frac{b_{2}}{v^{2}}\right) \sin \left(4 \pi v \tau_{\mathrm{He}}+\phi_{\mathrm{He}}\right) \\
& +\left(c_{1}+\frac{c_{2}}{v^{2}}\right) \sin \left(4 \pi v \tau_{\mathrm{BCZ}}+\phi_{\mathrm{BCZ}}\right) .
\end{aligned}
$$

Finally the amplitude $\bar{A}_{\mathrm{He}}$ of the helium-induced modulation was taken as the mean value of $b_{1}+\frac{b_{2}}{v^{2}}$ over the the range $[1.5,3] \mathrm{mHz}$, both quantities being scaled by $\Delta v / \Delta v_{\odot}$ (we take $\Delta v_{\odot}=135 \mu \mathrm{Hz}$ ). This last factor is introduced because the amplitude of the signal scales with the mean density, as does the large separation $\Delta v$ (see Basu et al. 2004). Figure 3 illustrates the results: a variation in helium fraction from 0.24 to

\footnotetext{
${ }^{5}$ Interestingly the $\theta^{1}$ Tau $\mathrm{B}$ star has a very similar mass $(1.28 \pm$ $0.13 M_{\odot}$ ) to that of Finsen $342 \mathrm{~B}$ so that our analysis also applies to this object.

${ }^{6}$ The region of the second ionization of helium has a non-negligible extent and one should use other fits than Eq. (5). However these fits provide similar helium fraction estimations as the one based on Eq. (5), see Basu et al. (2004).
}

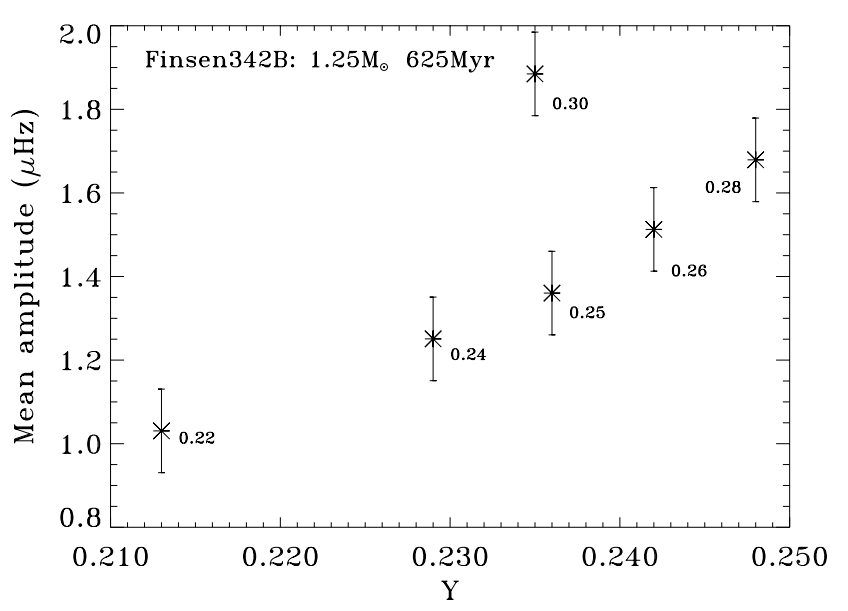

Fig. 3. The mean amplitudes of the second difference modulation induced through the second ionization of helium scaled by $\Delta v / \Delta v_{\odot}$. The means are given as function of helium fraction in the envelope at the age of the Hyades: $Y=0.213,0.229,0.236,0.242,0.248,0.235$ and for composition $\mathrm{C} 1$ (crosses). Because of the diffusion process these values do not correspond to the initial helium fractions that are indicated next to the crosses. Error bars of $0.1 \mu \mathrm{Hz}$ are only indicative.

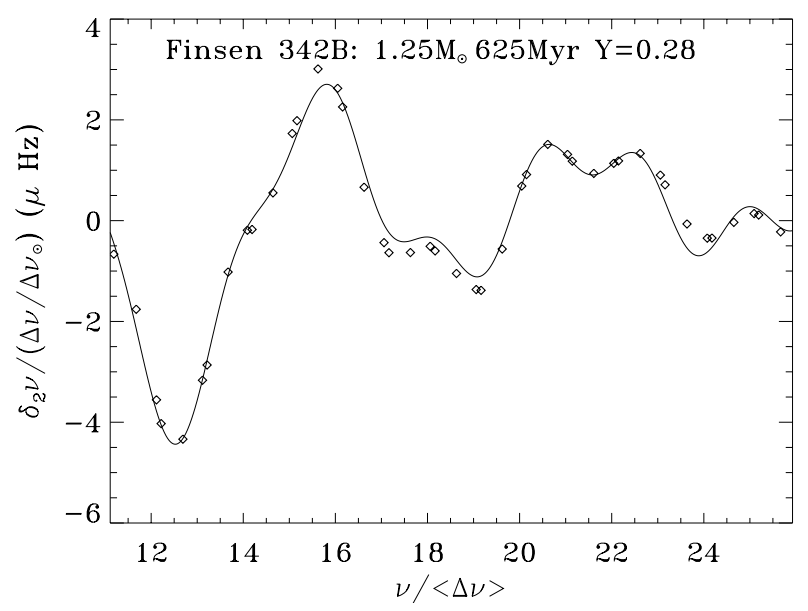

Fig. 4. Second difference and fit for Finsen 342 B if a helium fraction of $Y=0.28$ is assumed.

0.26 induces a change of $0.23 \mu \mathrm{Hz}$ in the fitted mean amplitude $\bar{A}_{\text {He }}$. Let us make here an important remark. In Hyades stars of $\approx 1.2 M_{\odot}$, diffusion is definitely not a negligible process because of the shallower outer convection zone. In the models at $625 \mathrm{Myr}$, the initial outer convective region helium fractions of 0.24 and 0.26 have decreased to 0.229 and 0.242 respectively. The $\Delta \bar{A}_{\mathrm{He}}$ variation is therefore larger than $0.1 \mu \mathrm{Hz}$ for $\Delta Y=0.01$. The diffusion effect becomes even more dramatic above $Y=0.28$ as the extension of the convection zone continuously diminishes with increasing helium fraction. The model that has an initial helium fraction of 0.3 indeed exhibits a lower Hyades age helium content $(Y=0.235)$ in its convection region as the model having initial helium fraction of 0.28 . In this instance we recall that the evolution of the $\bar{A}_{\mathrm{He}}$ amplitude is obviously not only related to the helium fraction but also depends on physical conditions in the region of helium second ionization. 


\subsection{Mass determination}

Binary systems have been extensively used to compute stellar masses on the basis of astrometric and spectroscopic measurements. A recent work of Söderhjehm (1999) based on Hipparcos astrometric data evaluates individual masses within 27 systems. For the subsample of 14 systems within the approximate mass range from 0.5 to $1.5 M_{\odot}$ the mean precision in mass determination is $0.11 M_{\odot}$ and exceeds 0.06 for all of the stars but two. In the Hyades cluster no system has its mass determined to better than $0.16 M_{\odot}$ and individual masses are available for only one system with a precision better than $0.2 M_{\odot}$. Making simultaneous use of spectroscopy and speckle interferometry can significantly improve the accuracy of such mass determinations: Pourbaix (2000) determines the masses to better than 5 percent for 25 stars from 40 binary systems. In very favourable cases the mass uncertainty has been decreased to slightly below $0.01 M_{\odot}$ (Latham et al. 1996). Combining observations of the Hyades binary system Finsen 342 (70 Tauri) over a period of $15 \mathrm{yr}$, Torres, Stefanik \& Latham (1997B) found $1.363 \pm 0.073 M_{\odot}$ and $1.253 \pm 0.075 M_{\odot}$ for the primary and the secondary components. However, because of the observational difficulties and the long amount of time required for careful interferometric and spectroscopic measurements only very few other stars in the Hyades (vB22, 51 Tau, $\theta^{2}$ Tau) have had their masses determined with similar precision (Torres Stefanik \& Latham 1997a; Peterson \& Solensky 1988).

Asteroseismology opens up new possibilities for accurate absolute or relative mass determinations. Its methods are not restricted to close binary systems but extend the determination of masses to all stars. We note by the way that the nonoccurrence of oscillations could be used as an indication of possibly unresolved multiple systems that are sometimes suspected (Söderhjelm 1999) in classical observations. In the following we focus on Hyades stars in the 0.77 to $1 M_{\odot}$ mass range.

The asymptotic theory of p-mode frequencies (Tassoul 1980) makes it possible to relate the large separation $\Delta v_{\ell, n}=v_{\ell, n}-v_{\ell, n-1}$ to the stellar acoustic radius $\tau_{R}$, namely:

$$
\frac{1}{2 \Delta v_{\ell, n}} \sim \int_{0}^{R} \frac{\mathrm{d} r}{c}=\tau_{R} .
$$

Table 3 shows the mean $\Delta v$ for masses varying from $\sim 0.77$ to $1 M_{\odot}$ and for possible ages and compositions of the Hyades. We compute $\Delta v$ as the average of $\Delta v_{\ell, n}$ over $\ell=0,1$ and 2 and for frequencies between 3.5 and $4.5 \mathrm{mHz}$. The lower limit is approximately the frequency of the mode of radial order $n=15$ and degree $\ell=0$ in a $0.77 M_{\odot}$ model. For higher mass stars this frequency corresponds to higher orders because of the decrease of the acoustic radius with mass. It therefore always lies above $n=10$ which keeps the explored domain far from possible radiatively induced oscillations (see Sect. 2). The other reason why we utilize frequencies in this range is that, as can be seen in Fig. 5, the large separation reaches the asymptotic regime above $\approx 3 \mathrm{mHz}$; thus its direct interpretation in terms of acoustic radius becomes all the more reliable. Finally we set an upper limit of $4.5 \mathrm{mHz}$ on the computation of the mean
Table 3. Stellar parameters for $0.769(\mathrm{vB} 22 \mathrm{~B}), 0.8,0.95$ and $1 M_{\odot}$ Hyades stars of composition C1. For $0.95 M_{\odot}$ results are given for various ages and compositions. Large separation computations are based on the mean of $\Delta v_{\ell, n}$ in the frequency range going 3500 to $4500 \mu \mathrm{Hz}$. The scatter in this mean always lies between 0.5 and $0.7 \mu \mathrm{Hz}$. Only low degree modes $l=0,1$ and 2 have been taken into account in the computations.

\begin{tabular}{lccccc}
\hline \hline Composition & $\begin{array}{c}\text { Mass } \\
\left(M_{\odot}\right)\end{array}$ & $\begin{array}{c}\text { Age } \\
(\mathrm{Myr})\end{array}$ & $\begin{array}{c}T_{\text {eff }} \\
(\mathrm{K})\end{array}$ & $m_{v}$ & $\begin{array}{c}\Delta v \\
(\mu \mathrm{Hz})\end{array}$ \\
\hline $\mathrm{C} 1$ & 0.769 & 625 & 4707 & 10.19 & 206.2 \\
$\mathrm{C} 1$ & 0.8 & 625 & 4835 & 9.92 & 199.1 \\
$\mathrm{C} 1$ & 0.95 & 625 & 5454 & 8.84 & 170.2 \\
$\mathrm{C} 2$ & 0.95 & 625 & 5375 & 8.93 & 171.5 \\
$\mathrm{C} 3$ & 0.95 & 625 & 5297 & 9.02 & 172.7 \\
$\mathrm{C} 4$ & 0.95 & 625 & 5612 & 8.70 & 167.0 \\
$\mathrm{C} 5$ & 0.95 & 625 & 5232 & 9.10 & 173.1 \\
$\mathrm{C} 1$ & 0.95 & 500 & 5451 & 8.84 & 170.8 \\
$\mathrm{C} 1$ & 0.95 & 700 & 5456 & 8.83 & 169.8 \\
$\mathrm{C} 1$ & 1.00 & 625 & 5637 & 8.54 & 160.8 \\
\hline
\end{tabular}

large separation in order to reduce the scatter in this mean. We preferentially select the models at $625 \mathrm{Myr}$ which has recently be estimated to be the Hyades age (Perryman et al. 1998). However we also extend the analysis to 500 and $700 \mathrm{Myr}$ to check for the impact of evolution slightly outside the claimed age uncertainties for the cluster $\approx 50 \mathrm{Myr}$. Calculations of the large separation of lower mass stars $\left(0.95 M_{\odot}\right)$ show its value to be extremely accurate. In the frequency range considered the large separations are extremely flat; the scatter is $\sim 0.5 \mu \mathrm{Hz}$ (Fig. 5). Moreover we find that the large separation experiences very small variations with age. The very slow evolution of stars having masses below a solar mass explains this stability. The $0.9 M_{\odot}$ star main sequence lifetime exceeds 14 Gyr (Schaller et al. 1992). We note that the large separation is also very stable with composition (for any conceivable variation of the metal or helium fraction). We report a $\Delta v$ variation of $\sim 5.7 \mu \mathrm{Hz}$ ie $3.4 \%$ of its total value for $\Delta Y$ as high as 0.04 . The different values for the helium fraction determined by Pinsonneault et al. (1998) $(Y=0.283)$ and Perryman et al. (1998) $(Y=0.26)$ lead to a $\Delta v$ variation of $3.2 \mu \mathrm{Hz}$. The variation with metallicity is similarly small. Going from composition $\mathrm{C} 1$ to $\mathrm{C} 5$ there is roughly 0.2 dex difference in metal content (iron group metals represent less than $8 \%$ of the global metal mass fraction). Then we note however that $\Delta v$ only changes by $2.9 \mu \mathrm{Hz}$. It is well known that metals mostly affect the stellar plasma opacity. The opacity in turn changes the stellar stratification in radiative zones but has a much weaker effect in outer convection zones where stratification is mostly adiabatic. Yet this is precisely the part of the star that mainly contributes to a large separation value. As illustrated by the aforementioned asymptotic expression the large separation is determined where the sound speed is at its lowest, i.e. in the coolest regions. In the $0.95 M_{\odot}$ star of $\mathrm{C} 1$ composition the convection zone represents $0.29 R_{\star}$ but conversely its acoustic radius is $0.59 \%$ of the total acoustic radius. For the 


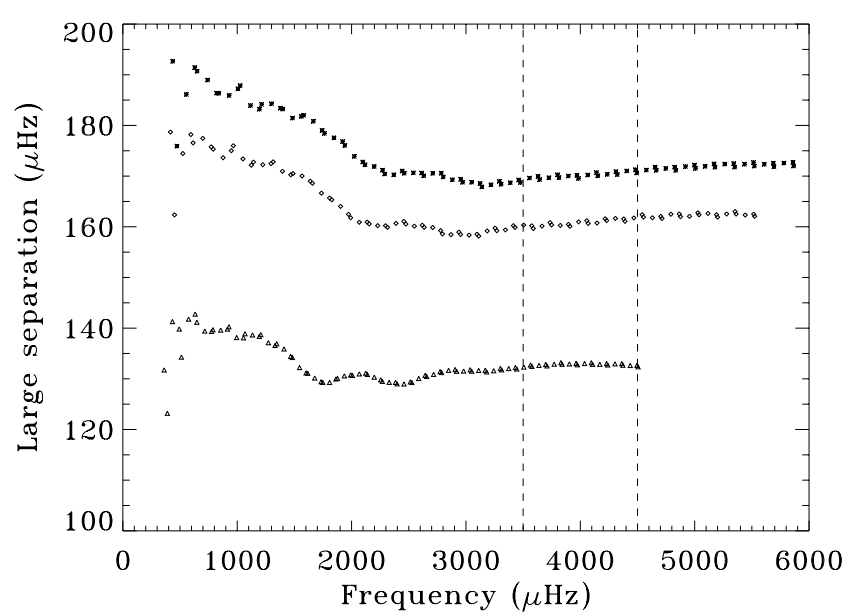

Fig. 5. Large separation as function of frequency and for different masses; crosses: $0.95 M_{\odot}$, diamonds: $1 . M_{\odot}$ and triangles: $1.15 M_{\odot}$ The vertical dashed lines shows the $3500-4500 \mu \mathrm{Hz}$ range over which we consider the mean large separation.

other compositions we check that these values are different by less than $3 \%$.

The age- and composition-related stability of $\Delta v$ make it easier for the effect of mass to show up. A change of $0.05 M_{\odot}$ gives rise to $\mathrm{a} \approx 10 \mu \mathrm{Hz}$ discrepancy. This variation clearly is much larger than the variations associated with mass or composition, and also larger than the resolution level of future spaceborne experiments such as EDDINGTON that should determine frequencies to the level of $0.3 \mu \mathrm{Hz}$ (Favata et al. 2000). Hence simple measurements of the large separation variation might be used for the mass determination of lower main sequence single Hyades stars. The accuracy in mass determination will reach a few percent of solar mass. As oscillations are expected for any solar-like star the sample of these objects having accurately determined masses will be greatly increased.

The predicted mass accuracy is comparable to or above the usual precision achieved when one relies on binary systems. It improves towards lower mass objects where the age determination uncertainties are negligible. However, as the mass decreases some other difficulties arise because of higher complexity in the input equation of state or low-temperature opacities (Chabrier \& Baraffe 1997). Besides this many aspects of the physics of convection in the outer regions of cool stars ( $\left.T_{\text {eff }}=4000-5000 \mathrm{~K}\right)$ remain to be investigated (Ludwig et al. 1999; 2002). A straightforward interpretation of the large separation in terms of mass might seem optimistic and it is perharps safer to use the large separation as an indicator of the mass hierarchy. By chance the vB22 system provides the best known mass in the Hyades: its secondary component $\left(0.769 \pm 0.005 M_{\odot}\right.$; Peterson \& Solensky 1988) lies in the region we have studied (Table 3).

\section{The extension of outer convection in the Hyades}

The precise extension of convective mixing in stars is crucial in several respects. First it has a direct impact on stellar evolution. Namely the occurrence of lower overshooting would significantly affect the evolution of low mass objects after they leave the main sequence and when they ascend the red giant branch (Alongi et al. 1991). The history of the surface abundances of the light elements closely depends on the extent of the convection zone. This in turn strongly constrains any inference on the dynamical phenomena in envelopes (Piau \& Turck-Chièze 2002; Piau et al. 2003). Finally convection and rotation remain the key ingredients for improving our knowledge of the angular momentum evolution or of the surface magnetic activity. In this instance observational studies of young stars are now capable of providing new clues on the precise nature of the dynamo process responsible for surface magnetic manifestations (Feigelson et al. 2003; Donati et al. 2003).

Thanks to the increase of computational power, direct three-dimensional modelling increasingly approches realistic physical conditions of stellar convection. Many of these studies have focused on solar-type outer convection zones (Brummell et al. 2002 and references therein) and now also begin to address convective cores (Browning et al. 2004). However, the corresponding convective flows are extremely swift and turbulent. Consequently it is not possible to model convection in its full complexity and a fortiori to model stellar evolution in the framework of such direct approaches. For almost half a century we have relied on parametrized theories for convection such as the mixing length theory (Böhm-Vitense 1958). As is well known the MLT possesses one main free parameter $\alpha_{\text {MLT }}$ that is empirically adjusted by means of measurements of the actual solar radius. Some studies of close binary systems suggest that no change of $\alpha_{\mathrm{MLT}}$ is required from the Sun to other low mass stars (Fernandes et al. 1998) while others suggest some slight changes $\approx 0.2$ with mass, e.g. in the $\alpha$ Cen system (Eggenberger et al. 2004). There are no basic physical reasons why this parameter should be independent of age, mass or composition.

Our goal here is to check how seismic information can be used to acquire better knowledge of convection. On the one hand the convective efficiency in the superadiabatic layers directly affects the extent of the convection zone. On the other hand seismology has provided direct measurements of the depth of the convection zone in the solar case (Christensen-Dalsgaard et al. 1991). It is therefore possible to relate seismic measurements to convective efficacy. Thanks to recently developed techniques one can address the issue of the depth of the outer convection zone in other stars than our Sun (e.g. Monteiro et al. 2000; Roxburgh \& Vorontsov 2001; Ballot et al. 2004, hereafter BTG04): the presence of a discontinuity in stellar structure is detectable through (low-degree) mode phase shifts where it induces oscillatory behaviour. For instance the second difference $\left(\delta_{2} v_{\ell, n}=v_{\ell, n-1}-2 v_{\ell, n}+v_{\ell, n+1}\right)$ exhibits modulations whose periodicities are directly related to the acoustic depth of the discontinuities (see Fig. 6). We quantitatively evaluate the variations of this parameter resulting from the convection / radiation discontinuity for various Hyades models in the 1 to $1.3 M_{\odot}$ range. This range lies above the masses addressed in binary systems by Fernandes et al. (1998) and is interesting to address in the Hyades in several respects. As open clusters comprise stars with marginally different compositions or ages, they offer the possibility to specifically check the impact of mass on the convective efficiency in the superadiabatic layers. Then the oscillations will be more easily 
detected above $\approx 1 M_{\odot}$ as the corresponding stars exihibit visual magnitudes below $\approx 8 m_{V}$ and show larger fluctuations than the Sun and their lower mass counterparts (cf. Sect. 2). At the same time a Hyades member of $\approx 1 M_{\odot}$ should have lost a significant fraction of its initial angular momentum and its rotation rate should be small all the way down to its center (Piau et al. 2003). This is an important point as the present analysis does not take into account rotationally induced effects on models and oscillation frequencies. In this instance Hyades observations suggest a rapid decrease of the equatorial velocity as a function of $T_{\text {eff }}$ between 7000 and $6000 \mathrm{~K}$. As suggested by Gaigé (1993) whereas below $T_{\text {eff }}=6400 \mathrm{~K}$ there are no stars with an equatorial velocity above $30 \mathrm{~km} \mathrm{~s}^{-1}$ the stars above $T_{\text {eff }}=7000 \mathrm{~K}$ exhibit a distribution with a large scatter from 0 up to $\approx 200 \mathrm{~km} \mathrm{~s}^{-1}$. For masses of $1,1.15$ and 1.3 $M_{\odot}$ we compute $T_{\text {eff }}=5640,6110,6500 \mathrm{~K}$ respectively at the age of the Hyades and the mass interval should therefore correspond to slow or moderate rotators.

Finally the mass interval considered covers the red wing of the so called lithium gap (Boesgaard \& Tripicco 1986): stars with the same age as the Hyades or older having effective temperatures between 6300 and $6850 \mathrm{~K}$ are lithiumand also beryllium-deficient in comparison to hotter or cooler stars. This is most probably related to a rotationally induced (magneto)hydrodynamic mixing process occurring at the top of the radiation region. For almost 20 years the lithium gap has been an active research field both theoretically (Talon \& Charbonnel 1998) and observationally (Boesgaard \& King 2002).

To determine the extent of the convection zone we make use of the second difference $\delta_{2} v_{\ell, n}$, exclusively evaluated from modes of degree $\ell=0,1$ and 2 . For the order $n$ we have considered the range between 13 and 26 which corresponds to the most excited modes in the solar case. Two periods are clearly seen in $\delta_{2} v_{\ell, n}$ vs. $v_{\ell, n}$ (see Fig. 6): the smallest oscillation is associated to the base of the convection zone (hereafter BCZ). The largest corresponds to the discontinuity induced by the second ionization region of helium which has been discussed in Sect. 3.1. To reduce its impact we subtract a smoothed interpolation of the raw data from $\delta_{2} v_{\ell, n}$. This procedure is more precisely described in BTG04. We then perform a spectral analysis to evaluate the acoustic depth of the convection zone $\tau_{\mathrm{BCZ}}$ (Fig. 6). The transform gives $\tilde{\tau}_{\mathrm{BCZ}}$ which is an estimate of $\tau_{\mathrm{BCZ}}$ biased by surface effects (Christensen-Dalsgaard et al. 1995). In the solar case there is for instance a $200 \mathrm{~s}$ difference between $\tau_{\mathrm{BCZ}}$ and $\tilde{\tau}_{\mathrm{BCZ}}$. Similarly half the inverse of the large separation, $\frac{1}{2 \Delta v}$, is shifted by this amount with respect to the acoustic radius $\tau_{R}$ (cf. Eq. (6)). The difference $\frac{1}{2 \Delta \nu}-\tilde{\tau}_{\mathrm{BCZ}}$ is therefore unbiased and corresponds to the acoustic radius of the convective base $\Delta \tau_{\mathrm{BCZ}}$ (BTG04).

$\Delta \tau_{\mathrm{BCZ}}=\tau_{R}-\tau_{\mathrm{BCZ}} \cong \frac{1}{2 \Delta v}-\tilde{\tau}_{\mathrm{BCZ}}$.

We use $\Delta \tau_{\mathrm{BCZ}}$ as it is not (or less) dependent than $\tilde{\tau}_{\mathrm{BCZ}}$ on surface effects where the physical description is less under control (turbulent pressure, superadiatic effects). Figure 7 shows the acoustic radii of convective bases $\Delta \tau_{\mathrm{BCZ}}$ scaled by $\Delta v / \Delta v_{\odot}$ as a function of effective temperatures and for the three $\alpha_{\mathrm{MLT}}$
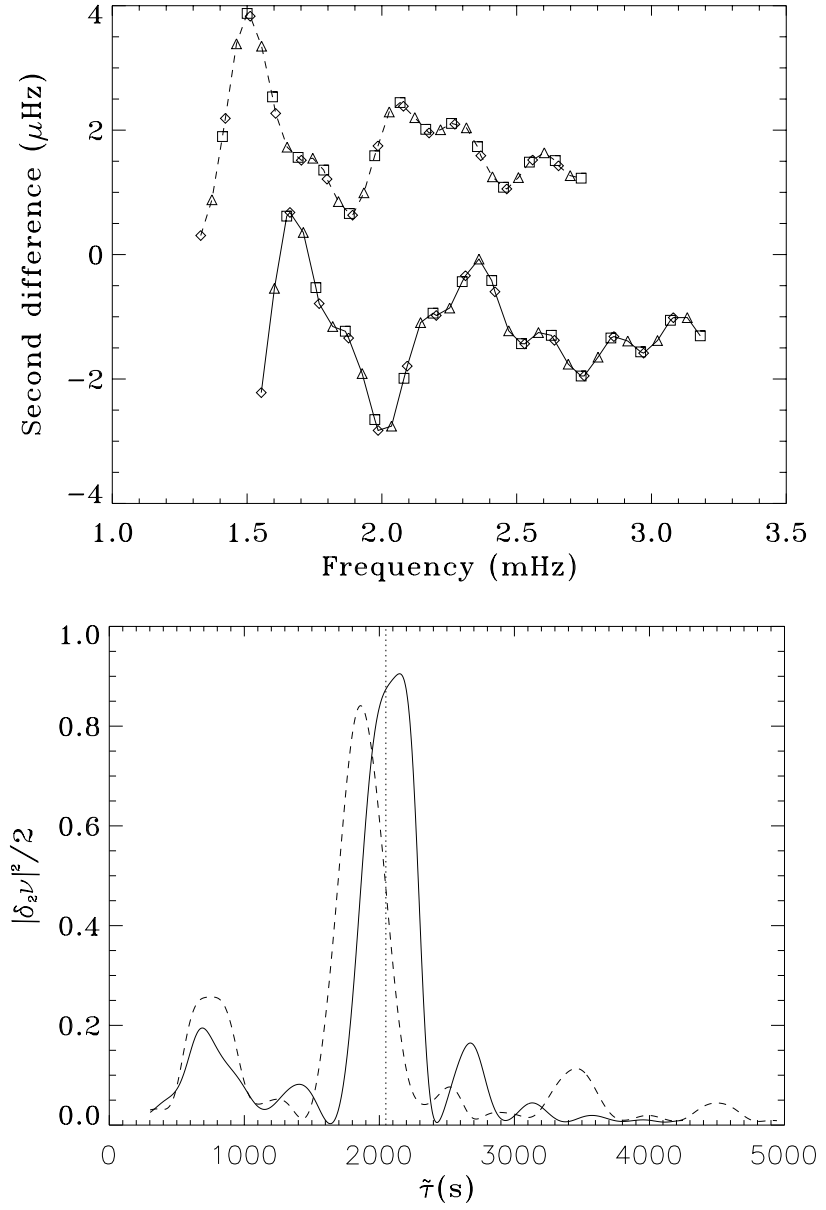

Fig. 6. Upper panel: the second difference as function of frequency for two Hyades models ( $625 \mathrm{Myr}$ ) of similar $T_{\text {eff }} \approx 6550 \mathrm{~K}$ but with different $\alpha_{\mathrm{MLT}}$ and masses. $\diamond: \ell=0, \Delta: \ell=1, \square: \ell=2$. Dashed line: $1.35 M_{\odot}$ and $\alpha_{\mathrm{MLT}}=1.566$. Continuous line: $1.3 M_{\odot}$ and $\alpha_{\mathrm{MLT}}=$ 1.966. In order to make the figure clearer the upper and lower tracks have been shifted by 1.5 and $-1.5 \mu \mathrm{Hz}$ respectively along the ordinate axis with respect to the original values. Lower panel: amplitudes for the Fourier transforms of second differences presented in the upper panel with similar conventions on linestyle. A smooth interpolation of second differences has been performed and subtracted to raw data before the Fourier transform in order to attenuate the He II ionization effect (this effect is still visible through the peaks around $800 \mathrm{~s}$ ). The vertical dashed line shows the middle of the continuous line peak at half maximum.

values $1.566,1.766$ (our solar calibrated value) and 1.966 . The acoustic radii scales with the mean density and inversely with frequencies. As in Fig. 3, the scaling factor $\Delta v / \Delta v_{\odot}$ therefore makes it possible to isolate the effect of the $\alpha_{\text {MLT }}$ parameter. It moreover reinforces our ability to determine changes in $\Delta \tau_{\mathrm{BCZ}}$ with effective temperature because it extends the variation range of $\Delta \tau_{\mathrm{BCZ}}$. The higher $\alpha_{\mathrm{MLT}}$ higher the convective efficiency and deeper the convection zone. As observed in Fig. 7 this in turn translates into smaller acoustic radii for the internal radiative regions. In Fig. 7 we plot a typical $\sigma\left(T_{\text {eff }}\right)$ error bar of $55 \mathrm{~K}$ for the effective temperature as estimated by Thorburn et al. (1993). The errors made in the determination of $\Delta \tau_{\mathrm{BCZ}}$ are extensively discussed in BTG04. We summarize the results here. Firstly there is a intrinsic error due to the method itself. 


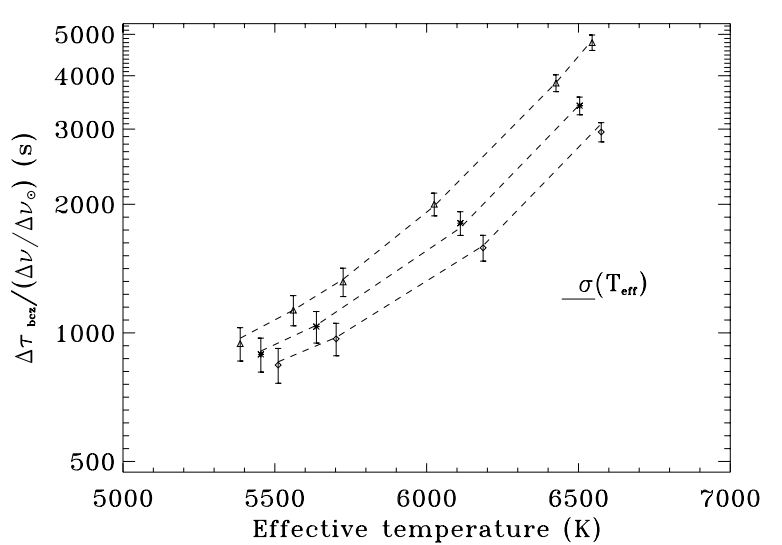

Fig. 7. Acoustic radii of the bases of the outer convection zones scaled by $\Delta v / \Delta v_{\odot}$ and as a function of the effective temperature at the age of the Hyades. Three $\alpha_{\text {MLT }}$ have been considered.The symbols show results extracted from $\delta_{2} v$. Triangles: $\alpha_{\mathrm{MLT}}=1.566$, masses $0.95,1$, $1.05,1.15,1.3$ and $1.35 M_{\odot}$; Stars: $\alpha_{\mathrm{MLT}}=1.766$, masses $0.95,1$, 1.15 and $1.3 M_{\odot}$. Diamonds: $\alpha_{\mathrm{MLT}}=1.966$, masses $0.95,1,1.15$ and $1.3 M_{\odot}$. The dashed lines indicate the true BCZ positions in the different models The horizontal error bar $\sigma\left(T_{\text {eff }}\right)$ is $2 \times 55 \mathrm{~K}$. The vertical error bars are discussed in the text.

This includes the effects of the limited number of points and the unknown residual bias of the estimator. For the modelled solarlike stars, this error is smaller than $1.5 \%$, and generally smaller than $1 \%$ in units of total stellar acoustic radius. This difference can be observed in Fig. 7 by comparing the symbol positions with respect to the dashed lines. Secondly the effects of the uncertainties on the frequencies can be estimated with Monte Carlo simulations. For expected errors of $0.1-0.15 \mu \mathrm{Hz}$ on the frequencies, the error induced in the determination of $\Delta \tau_{\mathrm{BCZ}}$ is around $2-2.5 \%$. Thus we have considered a pessimistic global error of $3 \%$ in units of total stellar acoustic radius.

Using a 2D hydrodynamic code including radiative transfer Ludwig et al. (1999) evaluate the stratification of the outer convection zone for various surface conditions in $T_{\text {eff }}$ and $\log g$. They cover stellar types from $\mathrm{K}$ to $\mathrm{F}\left(4300 \mathrm{~K} \leq T_{\text {eff }} \leq 7100 \mathrm{~K}\right.$ and $2.54 \leq \log g \leq 4.74)$. The $\alpha_{\text {MLT }}$ should vary from $\approx 1.3$ to $\approx 1.75$ to match the entropy jumps they find. The 0.4 range we consider here for $\alpha_{\text {MLT }}$ is therefore representative of the variations with surface conditions expected in convection efficiency. Interestingly Lebreton et al. (2001) find that Hyades isochrones having $\alpha_{\mathrm{MLT}}=1.6-1.8$ (respectively $\alpha_{\mathrm{MLT}} \lesssim 1.4$ ) better fit the $M_{V}-B-V$ observed magnitude diagram in the 1.3-1.7 $M_{\odot}$ range (respectively below $1 M_{\odot}$ ). We note here that owing to the errors any variation of convective efficiency with stellar type corresponding to an amplitude $\Delta \alpha_{\text {MLT }} \approx 0.2$ should be clearly observable in a diagram similar to Fig. 7.

To push the investigation further we have computed stellar models with variable $\alpha_{\text {MLT }}$ parameters. Following the Ludwig et al. (1999) scaling-law, $\alpha_{\text {MLT }}$ varies with the surface conditions ( $T_{\text {eff }}$ and $\log g$ ). With regard to constant (solar-calibrated) $\alpha_{\mathrm{MLT}}=1.76$ models the difference in $\Delta \tau_{\mathrm{BCZ}}$ for the variable $\alpha_{\text {MLT }}$ models is quite small ( $\$ 20 \mathrm{~s}$ ) except for the $1.3 M_{\odot}$ star where it reaches $95 \mathrm{~s}$. This stems from Ludwig's result that around solar conditions the $\alpha_{\text {MLT }}$ values exhibit a plateau in the $T_{\text {eff }}-\log g$ plane. The slightly larger $\Delta \tau_{\mathrm{BCZ}}(\approx 100 \mathrm{~s})$ of the
Table 4. Column 1: mass. Columns 2 and 3: surface effective temperature $T_{\text {eff }}$ and gravity $\log g$. Column 4: $\alpha_{\mathrm{MLT}}$, Col. 5: acoustic radius of the base of the outer convection zone $\Delta \tau_{\mathrm{BCZ}}$ as deduced from the Fourier transform of the second difference and typical error bars; Col. 6: specific entropy in the deep convective region $s_{\mathrm{cz}}$. The models constructed with a variable $\alpha_{\mathrm{MLT}}$ are to be found in the last section of the table. The reported $\alpha_{\mathrm{MLT}}$ values are those corresponding to the surface conditions at the age of the Hyades.

\begin{tabular}{lccccc}
\hline \hline $\begin{array}{l}\text { Mass } \\
\left(M_{\odot}\right)\end{array}$ & $\begin{array}{c}T_{\text {eff }} \\
(\mathrm{K})\end{array}$ & $\log g$ & $\alpha_{\mathrm{MLT}}$ & $\begin{array}{c}\Delta \tau_{\mathrm{BCZ}} \\
(\mathrm{s})\end{array}$ & $\begin{array}{c}s_{\mathrm{cZ}} \\
\left(\mathrm{erg} \mathrm{K}^{-1} \mathrm{~g}^{-1} / 10^{9}\right)\end{array}$ \\
\hline 0.95 & 5386 & 4.54 & 1.566 & $1155 \pm 122$ & 1.67 \\
1. & 5561 & 4.51 & 1.566 & $1304 \pm 120$ & 1.72 \\
1.05 & 5725 & 4.48 & 1.566 & $1426 \pm 152$ & 1.77 \\
1.15 & 6025 & 4.41 & 1.566 & $1892 \pm 157$ & 1.88 \\
1.3 & 6426 & 4.31 & 1.566 & $2934 \pm 198$ & 2.19 \\
1.35 & 6545 & 4.28 & 1.566 & $3394 \pm 194$ & 2.38 \\
\hline 0.95 & 5454 & 4.56 & 1.766 & $1123 \pm 121$ & 1.66 \\
1. & 5637 & 4.53 & 1.766 & $1232 \pm 118$ & 1.70 \\
1.15 & 6111 & 4.44 & 1.766 & $1777 \pm 165$ & 1.85 \\
1.3 & 6504 & 4.34 & 1.766 & $2697 \pm 206$ & 2.12 \\
\hline 0.95 & 5511 & 4.57 & 1.966 & $1085 \pm 120$ & 1.65 \\
1. & 5702 & 4.55 & 1.966 & $1185 \pm 119$ & 1.69 \\
1.15 & 6186 & 4.46 & 1.966 & $1609 \pm 130$ & 1.83 \\
1.3 & 6575 & 4.35 & 1.966 & $2419 \pm 216$ & 2.39 \\
\hline 0.95 & 5458 & 4.56 & 1.781 & $1120 \pm 121$ & 1.66 \\
1. & 5640 & 4.53 & 1.774 & $1209 \pm 130$ & 1.70 \\
1.05 & 5807 & 4.50 & 1.774 & $1387 \pm 142$ & 1.74 \\
1.15 & 6101 & 4.43 & 1.741 & $1769 \pm 164$ & 1.86 \\
1.3 & 6465 & 4.32 & 1.662 & $2792 \pm 193$ & 2.15 \\
\hline & & & & &
\end{tabular}

"variable" $\alpha_{\mathrm{MLT}} 1.3 M_{\odot}$ star reflects the decrease in convective efficiency towards higher $T_{\text {eff }}$ predicted by Ludwig et al. (1999). Let us stress in this example the ability of seismology to give constraints for the hydrodynamic convection simulations (Robinson et al. 2004).

Table 4 summarizes some quantities characterising the convection together with the corresponding stellar surface conditions $\left(T_{\text {eff }}\right.$ and $\log g$ ). We recall here that although $\alpha_{\text {MLT }}$ surely is the most important parameter within the MLT framework it is by no means the only one. There are indeed several formulations of the MLT that give different stratification for identical $\alpha_{\text {MLT }}$ values as illustrated by Ludwig et al. (2002). To specify the MLT formulation we use, we provide four other parameters (such as the surface to volume ratio for convective elements) in a short appendix. Like any other local approach of convection (eg Canuto \& Mazzitelli 1991), the MLT is simplistic and can be criticized on many points. Indeed various $\alpha_{\text {MLT }}$ parameters are required to match hydrodynamic computations for velocity or thermal stratification in the upper convection region (Ludwig et al. 2002). For these reasons, we provide a more meaningful physical quantity than $\alpha_{\mathrm{MLT}}$ : the specific entropy of the deep convection zone $s_{\mathrm{cz}}$. By entropy of the deep convection zone we mean the entropy level reached where convective motions 
become extremely close to adiabatic. $s_{\mathrm{cz}}$ is directly related to the extent of the convection zone and to the convective efficiency in superadiabatic layers. It may therefore be assigned an intermediate role between hydrodynamic computations and seismology.

We shall end this section with two caveats. Firstly and as noticed in the introduction no overshoot was considered here. Monteiro et al. (2000) show that such a phenomenon would increase the amplitude and the acoustic depth of the signal resulting from the BCZ discontinuity. Thus any interpretation of the extent of the outer convection zone in terms of convective efficiency or $\alpha_{\text {MLT }}$ parameter depends on the overshoot. We nevertheless note that 1) the actual helioseismic inferences on overshooting suggest that this phenomenon is moderate, Monteiro et al. (1994) find $l_{\mathrm{ov}}=0.07 H_{\mathrm{p}} ; 2$ ) hydrodynamic simulations of the solar convection zone similarly suggest rather moderate overshooting: below $0.11 H_{\mathrm{p}}$ (Brummell et al. 2002). The predicted movements moreover do not mix the stable region with enough efficiency to change its stratification significantly ${ }^{7}$ i.e. leave no seismic signature. Secondly the diffusion process has been taken into account following Michaud \& Proffitt (1993). Although we do not report it here in details we mention that the amplitude of the BCZ-induced oscillatory behaviour in $\delta_{2} v_{\ell, n}$ increases both with age and mass of our Hyades models. This is opposite to the comparable effects on frequencies reported in Monteiro et al. (2000). Microscopic diffusion is responsible for this difference. Monteiro et al. mostly addressed ZAMS stars where this process has no impact and was therefore not included in their computations. The description of diffusion we use is valid for $\mathrm{G}$ dwarfs and later types but becomes unrealistic for earlier types. Above $T_{\mathrm{eff}} \approx 6500 \mathrm{~K}$ the radiative diffusivity which we do not consider plays a non-negligible role (Morel \& Thevenin 2002). Besides this the stellar rotation increases and the microscopic diffusion interacts more strongly with one or several macroscopic mixing processes as it is suggested by the occurrence of the lithium dip.

\section{Summary and conclusion}

We computed classical and seismic stellar parameters using the stellar evolution code CESAM and the adiabatic pulsation package of Christensen-Dalsgaard. Classical and seismic variables were employed to constraint the stellar models. Only $\ell=0,1,2$ low-degree modes were considered. As a preliminary analysis we estimated apparent visual magnitudes, luminosity fluctuations and a few other interesting seismic variables in three nearby open clusters. We subsequently focused on stars within the closest and best-known of these: the Hyades. We predicted (Sect. 2) that the Hyades stars with masses around or above solar should exhibit oscillations detectable by the near-future seismic space-borne experiments. Future groundbased observations with HARPS or ESPaDOnS, in probing surface velocities could similarly begin to answer some of the questions mentioned here. Exploiting the predicted oscillation frequencies we had three goals: to set constraints on mass,

\footnotetext{
7 They represent therefore strictly speaking overshoot and not penetrative convection.
}

composition and structure. Let us briefly sum up our results on these points and conclude.

The mean value of the large separation $\Delta v$ in the 3.5 to $4.5 \mathrm{mHz}$ frequency range is a strong function of the mass: we calculated (Sect. 3.2) that $\mathrm{a} \approx 0.05 M_{\odot}$ mass change induces a $\approx 10 \mu \mathrm{Hz}$ variation in $\Delta v$. This is much higher than the $\Delta v$ variation expected from typical uncertainties in age or composition (Table 3). Asteroseismology will therefore allow the determination of absolute and relative masses in nearby open cluster and with competitive accuracy when compared to the best astrometric and spectrometric mass evaluations to date. However, at variance with these measurements seismology requires only short observation runs and is not limited to a very small sample of binaries.

Asteroseismology can shed new light on the composition of open clusters(Sect. 3.1). As main opacity contributors, metals strongly influence the effective temperature $T_{\text {eff }}$ and have a direct impact on the internal structure which affects the large separation $\Delta v$. Using Hyades models of different masses we have shown that the location of the $T_{\text {eff }}-\Delta v$ relation in the corresponding diagram provides a measurement of the global metal content. In particular, we are able to distinguish models buildt with solar-intermetallic ratios from models buildt with non-solar-intermetallic ratios. This is an important issue as we recall that 1 ) iron only represents $\approx 2 \%$ of the total metal content in number 2) there is a clear scatter in $[\mathrm{O} / \mathrm{Fe}]$ vs. $[\mathrm{Fe} / \mathrm{H}]$ in the Galactic disk (Edvardsson et al 1993). As is well known, the amplitude $\bar{A}_{\mathrm{He}}$ of the second difference $\delta_{2} v$ main modulation stems from the second ionization region of helium. Its use to infer helium fraction in the envelope however requires other stellar parameters such as mass and radius to be known (Basu et al. 2004). The FINSEN 342 star belongs to a Hyades binary system. Its mass and age are accurately known. Given these constraints, we showed that for this star a variation of the initial helium mass fraction from 0.26 to 0.28 would increase $\bar{A}_{\mathrm{He}}$ by $0.2 \mu \mathrm{Hz}$.

The main feature of structure of solar-like stars is the presence of a radiative core surrounded by a convective region. Using $\delta_{2} v$ modulations with frequency we have evaluated the acoustic radii of the bases of the convection zones (BCZ) for 1 to $1.3 M_{\odot}$ Hyades models and to an accuracy better than $3 \%$ of the total acoustic radius (Sect. 4). As, for a given effective temperature, the acoustic radius of the $\mathrm{BCZ}$ depends on convective efficiency, asteroseismology provides a possible tool for constraining $\alpha_{\mathrm{MLT}}$ (Fig. 7). As is well known, the mixing-length theory does not give a physically consistent picture of convection. Nevertheless, it is interesting to constrain $\alpha_{\text {MLT }}$ in stars as the ongoing hydrodynamic computations on convection make predictions for this parameter. Seismology therefore offers a means to check our calculations (and understanding) of convection in the superadiabatic layers. The considered mass range is interesting in several respects: it starts where the knowledge of our Sun can presumably be transposed and then extends in a domain where variations in convection efficiency are suspected through HR diagram analysis (Lebreton et al. 2001) or binary systems calibrations (Eggenberger et al. 2004). Moreover the interval covers the red wing of the lithium dip where additional mixing is at work in the radiation zone 
(Talon \& Charbonnel 1998). Setting constraints on convection depth in this range will not only shed new light on the convection efficacy in related stars but will also help constraining any deeper dynamical mixing phenomenon.

Because they comprise stars of similar age and composition, open cluster are unique laboratories for stellar physics. In this respect the Hyades have proved to be of primary importance. This importance is transposed to the asteroseismology field. In the Hyades it is possible to take advantage of collective effects and also to select some especially interesting binaries. In this work we presented various seismic tests that could be performed assuming today's accuracy on the measurement of oscillation frequencies. These tests clearly open up new opportunities in the field of stellar physics. Applied to the Hyades, asteroseismology will allow precise mass and composition determinations which are crucial for valuable astrophysical tests. It will furthermore enable measurements of the depths of outer convection zones which in turn will provide indications of convective efficiency in stars.

Acknowledgements. Laurent Piau is grateful to Reza Samadi, MarieJo Goupil, Ilidio Lopes and Dimitri Pourbaix for their help and for useful discussions. Laurent Piau is also extremly grateful to the referee, Mario Monteiro, for its rapid and efficient correction work that clearly helped to improve the paper. Laurent Piau is presently scientific collaborator to the Belgian FNRS.

\section{Appendix}

The MLT version we use relies on the following equations (Cox $\&$ Giuli 1968) where $f_{1}, f_{2}, f_{3}$ and $f_{4}$ are dimensionless free parameters each having a specific physical meaning:

$v^{2}=f_{1} \frac{\Lambda^{2} g \delta\left(\nabla-\nabla_{\mathrm{e}}\right)}{H_{\mathrm{p}}}$.

$v$ is the convection velocity. $f_{1}$ describes the dissipation of the work of buoyancy forces due to viscosity and we set it to $\frac{1}{8}$.

$F=f_{2} \frac{\rho c_{\mathrm{p}} v T \Lambda\left(\nabla-\nabla_{\mathrm{e}}\right)}{H_{\mathrm{p}}}$.

$F$ is the convective energy flux. $f_{2}$ is related to the proportion between upward and downward matter movements and is set to $\frac{1}{2}$.

$\Gamma=\frac{\nabla-\nabla_{\mathrm{e}}}{\nabla_{\mathrm{e}}-\nabla_{\mathrm{ad}}}=\frac{\rho c_{\mathrm{p}} v \tau_{\mathrm{e}}}{f_{3} \sigma T^{3}}\left(1+\frac{f_{4}}{\tau_{\mathrm{e}}^{2}}\right)$.

$\Gamma$ is the convection efficiency. $f_{3}$ is related to the surface $/ \Lambda$ volume ratio of convective elements and is set to 24. Finally $f_{4}$ is related to radiative transfer inside convective bubbles and is set to 3 . Most of the symbols in the preceding equations keep their usual meanings (eg $\Lambda=\alpha_{\mathrm{MLT}} H_{\mathrm{p}}$ is the mixing length) and were defined in Sect. 2. In addition: $T$ is the temperature; $\sigma$ is the Stefan-Boltzmann constant; $\nabla, \nabla_{\mathrm{e}}$ and $\nabla_{\mathrm{ad}}$ are the mean thermal gradient, the convective element thermal gradient and the adiabatic thermal gradient respectively; $\tau_{\mathrm{e}}$ is the Rosseland optical thickness of a convective element. The four dimensionless parameters are similar to the Böhm-Vitense (1958) formalism with the exception of $f_{4}$ which she assessed to be zero. As remarked by Henyey et al. (1965), however, the value of this parameter only has a marginal impact on the general description of convection through MLT. We have moreover directly checked that our approach and results indeed are similar to other computations done within the Böhm-Vitense formalism.

\section{References}

Alexander, D. R., \& Ferguson, J. W. 1994, ApJ, 437, 879

Alongi, M., Bertelli, G., Bressan, A., \& Chiosi, C. 1991, A\&A, 244, 95

Alonso, A., Arribas, S., \& Martinez-Roger, C. 1996, A\&A, 313, 873

Arribas, S., \& Martinez-Roger, C. 1988, A\&A, 206, 63

Asplund, M., Grevesse, N., Sauval, A. J., Allende Prieto, C., \& Kiselman D. 2004, A\&A, 417, 769

Baglin, A., Auvergne, M., Catala, C., and the COROT Team 2001, Proc. of the SOHO 10/GONG 2000 Workshop: Helio- and asteroseismology at the dawn of the millennium, 2-6 October 2000, ESA SP-464

Baglin, A., Auvergne, M., Barge, P. and the COROT Team 2002, Proc. of the First Eddington Workshop on Stellar Structure and Habitable Planet Finding, 11-15 June 2001, ESA SP-485 2002

Ballot, J., Turck-Chièze, S., \& García, R. A. 2004, A\&A, 423, 1051 (BTG04)

Basu, S., \& Antia, H. M. 1995, MNRAS, 276, 1402

Basu, S., Mazumdar, A., Antia, H. M., \& Demarque, P. 2004, MNRAS, 350, 277

Bessell, M. S., Castelli, F., \& Plez, B. 1998, A\&A, 333, 231

Boesgaard, A. M., \& Tripicco, M. J. 1986, ApJ 302, L49

Boesgaard, A. M., \& Friel, E. D. 1990, ApJ, 351, 467

Boesgaard, A. M., \& King, J. R 2002, ApJ, 565, 587

Böhm-Vitense, E. 1958, Z. Astrophys., 46, 108

Browning, M. K., Brun, A. S., \& Toomre, J. 2004, ApJ, 601, 512

de Bruijne, J. H. J., Hoogerwerf, R., \& de Zeeuw, P. T. 2001, A\&A, 367,111

Brummell, N. H., Clune, T. L., \& Toomre, J. 2002, ApJ, 570, 825

Brun, A. S., Turck-Chièze, S., \& Zahn, J. P. 1999, ApJ, 525, 1032

Canuto, V. M., \& Mazzitelli, I. 1991, ApJ, 370, 295

Chabrier, G., \& Baraffe, I. 1997, A\&A 327, 1039

Christensen-Dalgaard, J. 1982, MNRAS, 199, 735

Christensen-Dalgaard, J., 1988, A Hertzsprung-Russell Diagram for Stellar Oscillations, Adv. helio- and asteroseismology: IAU Symp. 123, 295

Christensen-Dalgaard, J., Gough, D. O., \& Thomson, M. J. 1991, ApJ, 378,413

Christensen-Dalgaard, J., Monteiro, M. J. P. F. G., \& Thompson, M. J. 1995, MNRAS, 276, 283

Cox, J. P., \& Giuli, R. T. 1968, Principles of stellar structure (Gordon \& Breach)

Donati, J.-F., Cameron, A. Collier, Semel, M., et al. 2003, MNRAS, 345,1145

Donati, J. F., Catala, C., \& Landstreet, J. D. 2004, sf2a, confE., 278D

Edvardsson, B., Andersen, J., Gustafsson, B., et al. 1993, A\&A, 275, 101

Eggen, O. J. 1998, AJ, 116, 1810

Eggenberger, P., Charbonnel, C., Talon, S., et al. 2004, A\&A, 417, 235

Favata, F. Roxburgh, I., \& Christensen- Dalsgaard, J. 2000, EDDINGTON, Assessment study report, ESA-SCI

Feigelson, E. D., Gaffney, J. A., III, Garmire, G., Hillenbrand, L. A., \& Townsley, L. 2003, ApJ, 584, 911

Fernandes, J., Lebreton, Y., Baglin, A., \& Morel, P. 1998, A\&A, 338, 455 
Gaigé, Y. 1993, A\&A, 269, 267

Garcia Lopez, R. J., Rebolo, R., Herrero, A., \& Beckman, J. E. 1993, ApJ, 412, 173

Goldreich, P., \& Keeley, D. A. 1977, ApJ, 212, 243

Goldreich, P., \& Kumar, P. 1990, ApJ, 363, 694

Henyey, L., Vardya, M. S., \& Bodenheimer, P. 1965, ApJ, 142, 841

Houdashelt, M. L., Bell, R. A., \& Sweigart, A. V. 2000, AJ, 119, 1448

Houdek, G., Balmforth, N. J., Christensen-Dalsgaard, J., \& Gough, D. O. 1999, A\&A, 351, 582

Iglesias, C. A., \& Rogers, F. J. 1996, ApJ, 464, 943

Izotov, Y. I., \& Thuan, T. X. 1998, ApJ, 500, 188

Kjeldsen, H., \& Bedding, T. R. 1995, A\&A, 293, 87

Latham, D. W, Nordstroem, B., Andersen, J., et al. 1996, A\&A, 314, 864

Lopes, I., Turck-Chièze, S., Michel, E., \& Goupil, M.-J. 1997, ApJ, 480, 794

Lebreton, Y. 2000, ARA\&A, 38, 35

Lebreton, Y., Fernandes, J., Lejeune, T. 2001, A\&A 374, 540

van Leeuwen, F. 1999, A\&A, 341, L71

Lejeune, T., Cuisinier, F., \& Buser, R. 1998, A\&AS, 130, 65

Ludwig, H. G., Freytag, B., \& Steffen, M. 1999, A\&A, 346, 111

Ludwig, H. G.,Allard, F., \& Hauschildt, P. H. 2002, A\&A, 395, 99

Michaud, G., Proffitt, C. R. 1993, Inside the stars, IAU Coll., 137, ASP Conf. Ser., ed. A. Baglin, \& W. W. Weiss, 40, 426

Michel, E., Hernandez, M. M., Houdek, G., et al. 1999, A\&A, 342, 153

Mihalas, D., 1978, Stellar atmospheres, 2nd ed. (Freeman and Cie)

Mihalas, D., Dappen, W., \& Hummer, D. G. 1988, ApJ, 331, 815

Monteiro, M. J. P. F. G., Christensen-Dalsgaard, J., \& Thompson, M. J. 1994, A\&A, 283, 247

Monteiro, M. J. P. F. G., Christensen-Dalsgaard, J., \& Thompson, M. J. 2000, MNRAS, 316, 165

Morel, P., van't Veer, C., Provost, J., et al. 1994, A\&A, 286, 91

Morel, P. 1997, A\&AS, 124, 597

Morel, P., \& Thevenin, F. 2002, A\&A, 390, 611

Paulson, D. B., Saar, S. H., Cochran, W. D., \& Hatzes, A. P. 2002, AJ, 124,572
Peimbert, M., Peimbert, A. 2000, The Light Elements and their Evolution, Proc. IAU Symp. 198, ed. L. da Silva, R. de Medeiros, $\&$ M. Spite

Pepe, F., Rupprecht, G., Avila, G., et al. 2003, SPIE, 4841, 1045

Perryman, M. A. C., Brown, A. G. A., Lebreton, Y., et al. 1998, A\&A, 331,81

Peterson, D. M., \& Solensky, R. 1988, ApJ, 333, 256

Piau, L., \& Turck-Chièze, S. 2002, ApJ, 566, 419

Piau, L., Randich, S., \& Palla, F. 2003, A\&A, 408, 1037

Pinsonneault, M. H., Stauffer, J., Soderblom, D. R., King, J. R., \& Hanson, R. B. 1998, ApJ, 504, 170

Pourbaix, D. 2000, A\&AS. Ser., 145, 219

Robichon, N., Arenou, F., Lebreton, Y., Turon, C., Mermilliod, J.C. 1999, In Harmonizing Cosmic Distances Scales in a PostHipparcos Era, ed. D. Egret, \& A. Heck, ASP Conf. Ser. 167, 72

Robinson, F. J., Demarque, P., Li, L. H., et al. 2004, MNRAS, 347, 1208

Rogers, F. J., Swenson, F. J., \& Iglesias, C. A. 1996, ApJ, 456, 902

Roxburgh, I. W., Vorontsov, S. V. 2001, MNRAS, 322, 85

Samadi, R., \& Goupil, M.-J. 2001, A\&A, 370, 136

Samadi, R., Goupil, M.-J., Houdek, G. 2002, A\&A, 395, 563

Samadi, R., Georgobiani, D., Trampedach, et al. 2004, A\&A, submitted

Schaller, G., Schaerer, D., Meynet, G., Maeder, A. 1992, A\&AS, 96, 269

Söderhjelm, S. 1999, A\&A, 341, 121

Stello, D., Nissen, P. E. 2001, 374, 105

Talon, S., \& Charbonnel, C. 1998, A\&A 335, 959

Tassoul, M. 1980, ApJS, 43, 469

Thorburn, J. A., Hobbs, L. M., Deliyiannis, Constantine P., \& Pinsonneault, M. H. 1993, ApJ, 415, 150

Torres, G., Stefanik, R. P., \& Latham, D. W. 1997a, ApJ, 474, 256

Torres, G., Stefanik, R. P., \& Latham, D. W. 1997b, ApJ, 479, 268

Walker, G., Matthews, J., Kuschnig, R., et al. 2003, PASP, 115, 1023 